\title{
ON DEFECTIVITY OF FAMILIES OF FULL-DIMENSIONAL POINT CONFIGURATIONS
}

\author{
CHRISTOPHER BORGER AND BENJAMIN NILL
}

(Communicated by Patricia Hersh)

\begin{abstract}
The mixed discriminant of a family of point configurations can be considered as a generalization of the $A$-discriminant of one Laurent polynomial to a family of Laurent polynomials. Generalizing the concept of defectivity, a family of point configurations is called defective if the mixed discriminant is trivial. Using a recent criterion by Furukawa and Ito we give a necessary condition for defectivity of a family in the case that all point configurations are full-dimensional. This implies the conjecture by Cattani, Cueto, Dickenstein, Di Rocco, and Sturmfels that a family of $n$ full-dimensional configurations in $\mathbb{Z}^{n}$ is defective if and only if the mixed volume of the convex hulls of its elements is 1 .
\end{abstract}

\section{INTRODUCTION}

Let us fix some notation. Throughout the paper, a configuration $A \subset \mathbb{Z}^{n}$ denotes a finite subset of $\mathbb{Z}^{n}$. We write $A_{0}+A_{1}:=\left\{a_{0}+a_{1}: a_{0} \in A_{0}, a_{1} \in A_{1}\right\}$ for the Minkowski sum of two configurations $A_{0}, A_{1} \subset \mathbb{Z}^{n}$. We denote by $e_{1}, \ldots, e_{n}$ the standard basis vectors in $\mathbb{Z}^{n}$ and in this context also set $e_{0}:=0 \in \mathbb{Z}^{n}$. Furthermore we denote by $\Delta_{k}:=\left\{e_{0}, e_{1}, \ldots, e_{k}\right\}$ the vertices of the standard unimodular simplex. The dimension of $A \subset \mathbb{Z}^{n}$ is the dimension of its affine hull (which we denote by aff $(A)$ ) as an affine subspace of $\mathbb{R}^{n}$ and is denoted by $\operatorname{dim}(A)$. We call $A$ full-dimensional if $\operatorname{dim}(A)=n$. We say that two configurations $A \subset \mathbb{Z}^{n}, B \subset \mathbb{Z}^{m}$ are isomorphic and denote this by $A \cong B$ if there is an affine lattice isomorphism of the ambient lattices aff $(A) \cap \mathbb{Z}^{n} \rightarrow \operatorname{aff}(B) \cap \mathbb{Z}^{m}$ mapping $A$ onto $B$. A lattice polytope that is isomorphic to a standard unimodular simplex is called unimodular simplex. If a lattice homomorphism $\varphi: \mathbb{Z}^{n} \rightarrow \mathbb{Z}^{m}$ is surjective, we call $\varphi$ a lattice projection. For convenience we use the notation $[m]:=\{0, \ldots, m\}$.

Let us recall the definition of the mixed discriminant (see $\left.\mathrm{CCD}^{+} 13\right)$. Consider a configuration $A \subset \mathbb{Z}^{n}$. We say that $f \in \mathbb{C}\left[x, x^{-1}\right]=\mathbb{C}\left[x_{1}, x_{1}^{-1}, \ldots, x_{n}, x_{n}^{-1}\right]$ is supported on $A$ if it is of the form

$$
f=\sum_{a \in A} c_{a} x^{a},
$$

Received by the editors October 22, 2019, and, in revised form, March 9, 2020.

2010 Mathematics Subject Classification. Primary 14M25, 52B20; Secondary 52A39, 13P15.

Key words and phrases. Mixed discriminant, A-discriminant, defectivity, Cayley polytopes, lattice polytopes.

This work was funded by the Deutsche Forschungsgemeinschaft (DFG, German Research Foundation) - 314838170, GRK 2297 MathCoRe.

The second author is an affiliated researcher with Stockholm University and was partially supported by the Vetenskapsrådet grant NT:2014-3991. 
with $c_{a} \in \mathbb{C}$ for all $a \in A$. We call an isolated solution $u \in\left(\mathbb{C}^{*}\right)^{n}$ for a system of Laurent polynomials $f_{0}(x)=\cdots=f_{k}(x)=0$ a non-degenerate multiple root if the gradients $\nabla f_{i}(u)$ are linearly dependent, while any $k$ of them are linearly independent. Now consider $A_{0}, \ldots, A_{k} \subset \mathbb{Z}^{n}$. Each polynomial $f_{i}$ supported on $A_{i}$ is of the form $f_{i}=\sum_{a \in A_{i}} c_{i, a} x^{a}$, and we define the discriminantal variety $\Sigma_{A_{0}, \ldots, A_{k}}$ as the closure of the set of coefficients $c_{i, a}$ such that the corresponding system of the Laurent polynomials $f_{i}$ has a non-degenerate multiple root. If $\Sigma_{A_{0}, \ldots, A_{k}}$ is a hypersurface, one defines the mixed discriminant $\Delta_{A_{0}, \ldots, A_{k}}$ to be the up-to-sign unique irreducible integral polynomial defining it. Otherwise, and this is the case we are going to be interested in, we set $\Delta_{A_{0}, \ldots, A_{k}}=1$ and call the set of configurations $A_{0}, \ldots, A_{k}$ defective.

In the specific case of a single configuration $A \subset \mathbb{Z}^{n}$ the mixed discriminant $\Delta_{A}$ agrees with the $A$-discriminant as introduced in [GKZ94. Let us recall the relation of defectivity of a point configuration to defectivity of projective varieties. Let $A=\left\{a_{0}, \ldots, a_{k}\right\} \subset \mathbb{Z}^{n}$ and denote by $X_{A} \subseteq \mathbb{P}^{k}$ the toric variety obtained as the closure of the image of the morphism

$$
\varphi_{A}:\left(\mathbb{C}^{*}\right)^{n} \rightarrow \mathbb{P}^{k} \quad t \mapsto\left[t^{a_{0}}: \ldots: t^{a_{k}}\right] .
$$

Then the variety $X_{A}^{*}$ projectively dual to $X_{A}$ is the same as the projectivization of the variety $\Sigma_{A}$. The dual defect $\delta_{X_{A}}$ of $X_{A}$ is defined as $\delta_{X_{A}}:=\operatorname{codim}\left(X_{A}^{*}\right)-1$, and the variety $X_{A}$ is called defective if $\delta_{X_{A}}>0$. In particular, $X_{A}$ is defective if and only if $A$ is a defective configuration, or equivalently, the degree of the $A$ discriminant is zero. The $A$-discriminant, especially its degree, has been studied intensively starting with the book [GKZ94]. We refer to the survey article Pie15] for background and references. In particular, a special focus has been on the question of defectivity when $A$ is the set of all lattice points of its convex hull ([DR06], CDR08, DDRP09, DN10, DNV12]). In more general situations, conditions for defectivity were given in [CC07, [DFS07, [Est10, [Ito15. In particular, a complete characterization in terms of so-called iterated circuits was presented by Esterov [Est10] and proven in [Est18] (see also [For19] for a more general version). Recently, a different characterization was obtained by Furukawa and Ito [FI16] phrased in terms of so-called Cayley sums (we refer the reader to Section 2 for the definition of Cayley sums).

The study of defectivity of a family of point configurations has so far been addressed in $\mathrm{CCD}^{+} 13$, DEK14, Est19 and, using a slightly different definition of defectivity of a family, in Est10. By the so-called Cayley trick, their defectivity can be reduced to defectivity of their Cayley sum if all point configurations are full-dimensional (see Theorem 3.1). Using the recent results by Furukawa and Ito, this allows us to deduce a necessary condition for defectivity of a family. For this, let us introduce some notation. For $A \subset \mathbb{Z}^{n}$ we denote by $\langle A-A\rangle$ the subgroup of $\mathbb{Z}^{n}$ generated by the set $\left\{a_{1}-a_{2}: a_{1}, a_{2} \in A\right\}$ and say that $A \subset \mathbb{Z}^{n}$ is spanning if $\langle A-A\rangle=\mathbb{Z}^{n}$. More generally we say that a family $A_{0}, \ldots, A_{k} \subset \mathbb{Z}^{n}$ is spanning if $\left\langle A_{0}-A_{0}\right\rangle+\cdots+\left\langle A_{k}-A_{k}\right\rangle=\mathbb{Z}^{n}$.

Theorem 1.1. Let $k \leq n$ and $A_{0}, \ldots, A_{k} \subset \mathbb{Z}^{n}$ be full-dimensional configurations that form a spanning family. If $A_{0}, \ldots, A_{k}$ is defective, then the convex hull of the Minkowski sum $A_{0}+\cdots+A_{k}$ does not have any interior lattice points, i.e.,

$$
\operatorname{int}\left(\operatorname{conv}\left(A_{0}+\cdots+A_{k}\right)\right) \cap \mathbb{Z}^{n}=\emptyset .
$$


As a consequence, we get the following result, which was conjectured in $\mathrm{CCD}^{+} 13$, where it was proven in the 2-dimensional case as well as under additional smoothness assumptions.

Corollary 1.2. Let $A_{0}, \ldots, A_{n-1} \subset \mathbb{Z}^{n}$ be a spanning family of full-dimensional configurations. Then $A_{0}, \ldots, A_{n-1}$ is defective if and only if it has mixed volume 1. In this case, $A_{0}, \ldots, A_{n-1}$ are all translates of the vertex set of the same unimodular simplex.

Proof. Clearly, having mixed volume one implies defectivity. By Theorem 1 in Hov78] (or Corollary 3.2 of [Nil20] the mixed volume of $\operatorname{conv}\left(A_{0}\right), \ldots, \operatorname{conv}\left(A_{n-1}\right)$ can be computed as

$$
1+\sum_{\emptyset \neq I \subseteq[n-1]}(-1)^{n-|I|}\left|\operatorname{int}\left(\operatorname{conv}\left(\sum_{i \in I} A_{i}\right)\right) \cap \mathbb{Z}^{n}\right| .
$$

If $A_{0}, \ldots, A_{n-1}$ is defective, Theorem 1.1 implies that $\operatorname{conv}\left(A_{0}+\cdots+A_{n-1}\right)$, and therefore (as all $A_{i}$ are full-dimensional) also $\operatorname{conv}\left(\sum_{i \in I} A_{i}\right)$ for any $I \subseteq$ $[n-1]$, has no interior lattice points. This shows that the mixed volume of $\operatorname{conv}\left(A_{0}\right), \ldots, \operatorname{conv}\left(A_{n-1}\right)$ is 1. The last statement follows from Proposition 2.7 of [CCD $\left.{ }^{+} 13\right]$ (see also [EG15]).

Remark 1.3. After the first version of this paper was made available, there was another proof of Corollary 1.2 given by Esterov (Corollary 3.23 in [Est19]). Esterov's result is more general in the sense that it only makes the weaker assumption of $A_{0}, \ldots, A_{n-1}$ forming a so-called irreducible family instead of all configurations being full-dimensional. However, it does not generalize Theorem 1.1, as it only treats the case of $k=n-1$. It would be interesting to investigate whether the assumption of full-dimensionality in Theorem 1.1 can always be replaced by irreducibility of the family. We call a family $A_{0}, \ldots, A_{k} \subset \mathbb{Z}^{n}$ irreducible if no $l$ distinct members can be shifted to a common $(l+(n-1-k))$-dimensional affine subspace for any $l \in\{1, \ldots, k\}$.

Note that for given $A_{0}, \ldots, A_{k} \subset \mathbb{Z}^{n}$ one may always choose a spanning family whose mixed discriminantal variety equals $\Sigma_{A_{0}, \ldots, A_{k}}$. By applying a suitable transformation, this implies the following slightly more general version of Theorem 1.1.

Corollary 1.4. Let $k \leq n$ and let $A_{0}, \ldots, A_{k} \subset \mathbb{Z}^{n}$ be full-dimensional configurations. Define by $\Lambda:=\left\langle A_{0}-A_{0}\right\rangle+\cdots+\left\langle A_{k}-A_{k}\right\rangle$ the lattice spanned by these configurations. If $A_{0}, \ldots, A_{k}$ is defective, then

$$
\operatorname{int}\left(\left(A_{0}-a_{0}\right)+\cdots+\left(A_{k}-a_{k}\right)\right) \cap \Lambda=\emptyset,
$$

for all choices $a_{0}, \ldots, a_{k}$ such that $a_{i} \in A_{i}$ for all $i \in[k]$.

Remark 1.5. The statement of Theorem 1.1 is in general not true if we do not pose sufficient restrictions on the dimensions of the configurations. A counterexample is provided by choosing $A_{0}, A_{1} \subset \mathbb{Z}^{2}$ as

$$
A_{0}=\left(\begin{array}{lll}
0 & 1 & 2 \\
0 & 0 & 0
\end{array}\right) \text { and } A_{1}=\left(\begin{array}{lll}
0 & 0 & 0 \\
0 & 1 & 2
\end{array}\right) .
$$

It is straightforward to verify that the corresponding system

$$
f_{0}=c_{0,00}+c_{0,10} x_{1}+c_{0,20} x_{1}^{2}, f_{1}=c_{1,00}+c_{1,01} x_{2}+c_{1,02} x_{2}^{2}
$$


does not have a non-degenerate multiple root for any choice of coefficients. Therefore the variety $\Sigma_{A_{0}, A_{1}}$ is empty, in particular $A_{0}, A_{1}$ is a defective family, while $\operatorname{conv}\left(A_{0}+A_{1}\right)$ contains $(1,1)$ as an interior lattice point.

Remark 1.6. Note that the criterion for defectivity given in Theorem 1.1 is not sufficient. An easy class of counterexamples is given for $k=0$ by $A_{0}:=\operatorname{conv}\left(n \Delta_{n}\right) \cap$ $\mathbb{Z}^{n}$ for $n>1$. Clearly conv $\left(A_{0}\right)$ does not have any interior lattice points but cannot be defective since its lattice width is $n>1$.

Organization of the paper. In Section 2 we introduce Cayley sums and recall some basic results. Section 3 contains the proof of Theorem 1.1.

\section{Basics of CAyley sums}

As Cayley sums are going to play a crucial role in our proof, let us recall some basic facts.

Definition 2.1. Let $A_{0}, \ldots, A_{k} \subset \mathbb{Z}^{n}$ be configurations. We define the Cayley sum $A_{0} * \cdots * A_{k}$ as

$$
A_{0} * \cdots * A_{k}:=\left(A_{0} \times\left\{e_{0}\right\}\right) \cup\left(A_{1} \times\left\{e_{1}\right\}\right) \cup \cdots \cup\left(A_{k} \times\left\{e_{k}\right\}\right) \subset \mathbb{Z}^{n+k} .
$$

We call a Cayley sum $A_{0} * \cdots * A_{k}$ proper if all $A_{i}$ are non-empty. In this case one has $\operatorname{dim}\left(A_{0} * \cdots * A_{k}\right)=\operatorname{dim}\left(A_{0}+\cdots+A_{k}\right)+k$.

Let $F \subseteq A$ be a subconfiguration of a configuration $A \subset \mathbb{Z}^{n}$. We denote by $F^{c}=\{x \in A: x \notin F\}$ the complement of $F$ in $A$. Furthermore, we call $F$ a face of $A$ if it is the intersection of a face of the lattice polytope $\operatorname{conv}(A)$ with $A$ and denote by $\mathcal{F}(A)$ the set of all faces of $A$. We call a face $F \in \mathcal{F}(A)$ proper if $F \neq A$.

Definition 2.2. Let $A \subset \mathbb{Z}^{n}$ and $F_{0}, \ldots, F_{k} \in \mathcal{F}(A)$ be faces that cover $A$. We say that $F_{0}, \ldots, F_{k}$ form a Cayley decomposition of $A$ if there exists a lattice projection $\pi: \mathbb{Z}^{n} \rightarrow \mathbb{Z}^{k}$ such that $\pi\left(F_{i}\right) \subseteq\left\{e_{i}\right\}$ for all $i \in[k]$.

Remark 2.3. Clearly, a Cayley sum $A_{0} * \cdots * A_{k}$ has a Cayley decomposition into the faces $\left(A_{0} \times\left\{e_{0}\right\}\right), \ldots,\left(A_{k} \times\left\{e_{k}\right\}\right)$, and we denote them by $\tilde{A}_{i}:=A_{i} \times\left\{e_{i}\right\}$.

Proposition 2.4. Let $A \subset \mathbb{Z}^{n}$ be a configuration. Then the following are equivalent.

(1) There exists a Cayley decomposition of $A$ into non-empty faces $F_{0}, \ldots, F_{k}$ $\in \mathcal{F}(A)$.

(2) There exists a lattice projection $\pi: \mathbb{Z}^{n} \rightarrow \mathbb{Z}^{k}$ with $\pi(A)=\Delta_{k}$.

(3) There exist configurations $A_{0}, \ldots, A_{k} \subset \mathbb{Z}^{n-k}$ such that $A \cong A_{0} * \cdots * A_{k}$.

The proof is left to the reader (cf. [BN08, Proposition 2.3]).

Remark 2.5. Let $A \subset \mathbb{Z}^{n}$ be a configuration, let $F_{0}, \ldots, F_{k} \in \mathcal{F}(A)$ be a Cayley decomposition of $A$, and let $F \in \mathcal{F}(A)$ be an arbitrary face. Then we have a Cayley decomposition

$$
F \cong\left(F_{0} \cap F\right) * \cdots *\left(F_{k} \cap F\right) .
$$

In particular, any face of a Cayley sum $A_{0} * \cdots * A_{k}$ is isomorphic to a Cayley sum of (maybe empty) faces of each of the $A_{i}$.

Definition 2.6. Let $A_{0}, \ldots, A_{k} \subset \mathbb{Z}^{n}$ be configurations. We say that the Cayley sum $A_{0} * \cdots * A_{k}$ is of join type if the homomorphism $\left\langle A_{0}-A_{0}\right\rangle \oplus \cdots \oplus\left\langle A_{k}-A_{k}\right\rangle \rightarrow$ $\left\langle A_{0}-A_{0}\right\rangle+\cdots+\left\langle A_{k}-A_{k}\right\rangle \subset \mathbb{Z}^{n}$ given by $\left(a_{0}, \ldots, a_{k}\right) \mapsto a_{0}+\cdots+a_{k}$ is injective. 
Remark 2.7. As $\operatorname{dim}\left(\left\langle A_{0}-A_{0}\right\rangle \oplus \cdots \oplus\left\langle A_{k}-A_{k}\right\rangle\right)=\operatorname{dim}\left(A_{0}\right)+\cdots+\operatorname{dim}\left(A_{k}\right)$ and $\operatorname{dim}\left(\left\langle A_{0}-A_{0}\right\rangle+\cdots+\left\langle A_{k}-A_{k}\right\rangle\right)=\operatorname{dim}\left(A_{0}+\cdots+A_{k}\right)$, a Cayley sum $A_{0} * \cdots * A_{k}$ is of join type if and only if $\operatorname{dim}\left(A_{0}\right)+\cdots+\operatorname{dim}\left(A_{k}\right)=\operatorname{dim}\left(A_{0}+\cdots+A_{k}\right)$. In particular, the dimension of a proper Cayley sum $A_{0} * \cdots * A_{k}$ of join type equals $\operatorname{dim}\left(A_{0}\right)+\cdots+\operatorname{dim}\left(A_{k}\right)+k$, which is the maximal Cayley dimension for given dimensions of the summands $A_{0}, \ldots, A_{k}$.

\section{Proof of MAIN TheOREM}

The following result was presented by Di Rocco in a talk in June 2016 at the Fields Institute for Research in Mathematical Sciences and is soon to appear in an announced paper by Di Rocco, Dickenstein, and Morrison DDRM18 (see also $\mathrm{CCD}^{+} 13$ for the special case where $k=n-1$ ).

Theorem 3.1. If a family of configurations $A_{0}, \ldots, A_{k} \subset \mathbb{Z}^{n}$ is defective, then the Cayley sum $A_{0} * \cdots * A_{k} \subset \mathbb{Z}^{n+k}$ is defective.

This identification allows us to apply the following characterization of defective configurations by Furukawa and Ito [FI16] as the main tool in proving our statement about defectivity of a family of configurations.

Theorem 3.2 (Furukawa, Ito). Let $A \subset \mathbb{Z}^{n}$ be a spanning configuration. Then $A$ is defective if and only if there exist natural numbers $c<r$ and a lattice projection $\pi: \mathbb{Z}^{n} \rightarrow \mathbb{Z}^{n-c}$ such that $\pi(A) \cong B_{0} * \cdots * B_{r}$ where this Cayley sum $B_{0} * \cdots * B_{r}$ is of join type and $B_{i} \neq \emptyset$ for all $i \in[r]$.

It is a straightforward computation to show that $A_{0}, \ldots, A_{k} \subset \mathbb{Z}^{n}$ form a spanning family if and only if their Cayley sum $A_{0} * \cdots * A_{k} \subset \mathbb{Z}^{n+k}$ is spanning.

The following technical lemma is crucial for the proof of the main theorem.

Lemma 3.3. Let $A_{0}, \ldots, A_{k} \subset \mathbb{Z}^{n}$ be full-dimensional configurations and let $B_{0}, \ldots, B_{r} \subset \mathbb{Z}^{n+k-r}$ be non-empty configurations such that

$$
A_{0} * \cdots * A_{k} \cong B_{0} * \cdots * B_{r} \subset \mathbb{Z}^{n+k} .
$$

(a) One has $\operatorname{dim}\left(B_{i}\right) \geq \min (k, n)$ for all $i \in[r]$.

(b) If furthermore $\operatorname{dim}\left(B_{i}\right)<n$ for all $i \in[r]$, also the following inequality holds:

$$
\operatorname{dim}\left(B_{0}\right)+\cdots+\operatorname{dim}\left(B_{r}\right) \geq n-r+(r+1) k .
$$

Proof. For $k=0$ or $r=0$ one can directly verify that both statements hold. So we may assume that $k, r \geq 1$ and observe that in this case each of the $\tilde{B}_{i} \subseteq$ $B_{0} * \cdots * B_{r}$ (see Remark 2.3 is isomorphic to a proper face $B_{i}^{\prime} \subseteq A_{0} * \cdots * A_{k}$ and $B_{0}^{\prime}, \ldots, B_{r}^{\prime}$ form a Cayley decomposition of $A_{0} * \cdots * A_{k}$ (since the $\tilde{B}_{i}$ form a Cayley decomposition of $\left.B_{0} * \cdots * B_{r}\right)$. The complement $\left(B_{i}^{\prime}\right)^{c}$ of each of the $B_{i}^{\prime}$ is again a proper face of $A_{0} * \cdots * A_{k}$ (since this is true for the complement of $\tilde{B}_{i}$ ). Now let $i \in[r]$ be arbitrary and assume that $\operatorname{dim}\left(B_{i}\right)<n$ (otherwise (a) is trivial). Then $B_{i}^{\prime}$ cannot contain $\tilde{A}_{j}$ for any $j \in[k]$ and $\left(B_{i}^{\prime}\right)^{c}$ has non-empty intersection with each of the $\tilde{A}_{j}$. Therefore by Remark 2.5 in particular $\operatorname{dim}\left(B_{i}^{\prime}\right)^{c} \geq \operatorname{dim}\left(\left(B_{i}^{\prime}\right)^{c} \cap \tilde{A}_{j}\right)+k$ for all $j \in[k]$. Now if $\left(B_{i}^{\prime}\right)^{c}$ contained one of the $\tilde{A}_{j}$, this inequality would imply that $\operatorname{dim}\left(B_{i}^{\prime}\right)^{c} \geq n+k$ in contradiction to $\left(B_{i}^{\prime}\right)^{c}$ being a proper face of $A_{0} * \cdots * A_{k}$. 
So also $B_{i}^{\prime}$ has non-empty intersection with all of the $\tilde{A}_{j}$, and by Remark 2.5 we have

$$
B_{i}^{\prime} \cong\left(\tilde{A}_{0} \cap B_{i}^{\prime}\right) * \cdots *\left(\tilde{A}_{k} \cap B_{i}^{\prime}\right)
$$

which implies that

$$
\operatorname{dim}\left(\tilde{A}_{j} \cap B_{i}^{\prime}\right) \leq \operatorname{dim}\left(B_{i}^{\prime}\right)-k
$$

for all $j \in[k]$ and all $i \in[r]$ with $\operatorname{dim}\left(B_{i}\right)<n$. This in particular implies that $\operatorname{dim}\left(B_{i}\right)=\operatorname{dim}\left(B_{i}^{\prime}\right) \geq k \geq \min (k, n)$. Moreover, since the $B_{i}^{\prime}$ also form a Cayley decomposition of $A_{0} * \cdots * A_{k}$, we obtain

$$
\tilde{A}_{j} \cong\left(\tilde{A}_{j} \cap B_{0}^{\prime}\right) * \cdots *\left(\tilde{A}_{j} \cap B_{r}^{\prime}\right),
$$

and therefore assuming $\operatorname{dim}\left(B_{i}\right)<n$ for all $i \in[r]$, applying (11) yields

$$
\begin{aligned}
n=\operatorname{dim}\left(\tilde{A}_{j}\right) & \leq r+\operatorname{dim}\left(\tilde{A}_{j} \cap B_{0}^{\prime}\right)+\cdots+\operatorname{dim}\left(\tilde{A}_{j} \cap B_{r}^{\prime}\right) \\
& \leq r+\operatorname{dim}\left(B_{0}^{\prime}\right)-k+\cdots+\operatorname{dim}\left(B_{r}^{\prime}\right)-k .
\end{aligned}
$$

Note that the result above remains true in the more general setting of point configurations in $\mathbb{R}^{n}$ and the notion of isomorphy induced by affine bijections.

Let us recall that the codegree $\operatorname{codeg}(P)$ of a lattice polytope $P \subset \mathbb{R}^{n}$ is the smallest natural number $c \geq 1$ such that $\operatorname{int}(c P) \cap \mathbb{Z}^{n} \neq \emptyset$ (see e.g. [DN10]).

Proof of Theorem 1.1. As remarked above, Theorem 3.1 implies that $A_{0} * \cdots * A_{k} \subset$ $\mathbb{Z}^{n+k}$ is a spanning defective configuration. By Theorem 3.2 there exist $c<r$ and a lattice projection $\pi: \mathbb{Z}^{n+k} \rightarrow \mathbb{Z}^{n+k-c}$ such that $\pi\left(A_{0} * \cdots * A_{k}\right)$ has a Cayley decomposition of join type into non-empty faces $F_{0}, \ldots, F_{r} \in \mathcal{F}\left(\pi\left(A_{0} * \cdots * A_{k}\right)\right)$. Let us assume that $\operatorname{conv}\left(A_{0}+\cdots+A_{k}\right)$ has interior lattice points. By the wellknown connection between Cayley sums and weighted Minkowski sums (see e.g. [HRS00 $)$ this is equivalent to $(k+1) \cdot \operatorname{conv}\left(A_{0} * \cdots * A_{k}\right)$ having an interior point in $\mathbb{Z}^{n+k}$, which implies that $\operatorname{codeg}\left(\operatorname{conv}\left(A_{0} * \cdots * A_{k}\right)\right) \leq k+1$. By Proposition 2.4 we have a projection $\pi_{r}: \mathbb{Z}^{n+k-c} \rightarrow \mathbb{Z}^{r}$ that maps $\pi\left(A_{0} * \cdots * A_{k}\right)$ surjectively onto $\Delta_{r}$. Since under lattice projections the codegree of a lattice polytope cannot increase we get inequalities

$$
k+1 \geq \operatorname{codeg}\left(A_{0} * \cdots * A_{k}\right) \geq \operatorname{codeg}\left(F_{0} * \cdots * F_{r}\right) \geq \operatorname{codeg}\left(\Delta_{r}\right)=r+1 \text {, hence }
$$

$$
k \geq r .
$$

We observe that the lifts

$$
\hat{F}_{i}:=\pi^{-1}\left(F_{i}\right) \cap\left(A_{0} * \cdots * A_{k}\right)
$$

define a Cayley decomposition (in general not of join type) of $A_{0} * \cdots * A_{k}$. As $\pi$ is a projection of codimension $c$, we see that

$$
\operatorname{dim}\left(\hat{F}_{i}\right) \leq \operatorname{dim}\left(F_{i}\right)+c,
$$


for all $i \in[r]$. Combining this with the fact that the $F_{i}$ form a Cayley decomposition of join type and using Remark 2.7 one obtains

$$
\begin{aligned}
\operatorname{dim}\left(\hat{F}_{0}\right)+\cdots+\operatorname{dim}\left(\hat{F}_{r}\right) & \leq \operatorname{dim}\left(F_{0}\right)+\cdots+\operatorname{dim}\left(F_{r}\right)+c(r+1) \\
& =\operatorname{dim}\left(F_{0}+\cdots+F_{r}\right)+c(r+1) \\
& =n+k-c-r+c(r+1) \\
& =n+k+r(c-1) .
\end{aligned}
$$

Let us assume that $\operatorname{dim}\left(\hat{F}_{j}\right) \geq n$ for some $j \in[r]$. Therefore $\operatorname{dim}\left(F_{j}\right) \geq n-c$. Without loss of generality let $j=0$. As the $F_{i}$ form a Cayley decomposition of join type of the $(n+k-c)$-dimensional configuration $\pi\left(A_{0} * \cdots * A_{k}\right)$ we have the following inequality for the remaining summands:

$$
\begin{aligned}
\operatorname{dim}\left(F_{1}\right)+\cdots+\operatorname{dim}\left(F_{r}\right) & =\operatorname{dim}\left(F_{0}+\cdots+F_{r}\right)-\operatorname{dim}\left(F_{0}\right) \\
& =n+k-c-r-\operatorname{dim}\left(F_{0}\right) \\
& \leq n+k-c-r-(n-c) \\
& =k-r .
\end{aligned}
$$

However, on the other hand Lemma 3.3) (amplies that $\operatorname{dim}\left(\hat{F}_{i}\right) \geq k$ for all $i \in[r]$ (since we assumed $k \leq n$ ). So by (3) we have $\operatorname{dim}\left(F_{i}\right) \geq k-c$, which yields another inequality for the remaining summands:

$$
\operatorname{dim}\left(F_{1}\right)+\cdots+\operatorname{dim}\left(F_{r}\right) \geq r(k-c) .
$$

These inequalities contradict each other since $r(k-c)>k-r$, which can be seen by observing that $r$ is strictly positive and $c$ is strictly smaller than $r$.

Therefore $\operatorname{dim}\left(\hat{F}_{j}\right)<n$ for all $j \in[r]$. So we may apply part (b) of Lemma 3.3 and obtain $n-r+(r+1) k \leq \operatorname{dim}\left(\hat{F}_{0}\right)+\cdots+\operatorname{dim}\left(\hat{F}_{r}\right)$. Hence,

$$
n-r+(r+1) k \leq n+k+r(c-1),
$$

which is (since $r$ is strictly positive) equivalent to $k \leq c<r$, a contradiction.

\section{ACKNOWLEDGMENTS}

We thank Alicia Dickenstein and Sandra Di Rocco for fruitful discussions and for sharing their notes about Theorem 3.1. We furthermore thank Alexander Esterov for his interest and helpful comments.

\section{REFERENCES}

[BN08] Victor Batyrev and Benjamin Nill, Combinatorial aspects of mirror symmetry, Integer points in polyhedra-geometry, number theory, representation theory, algebra, optimization, statistics, Contemp. Math., vol. 452, Amer. Math. Soc., Providence, RI, 2008, pp. 35-66, DOI 10.1090/conm/452/08770. MR2405763

[CC07] Raymond Curran and Eduardo Cattani, Restriction of A-discriminants and dual defect toric varieties, J. Symbolic Comput. 42 (2007), no. 1-2, 115-135, DOI 10.1016/j.jsc.2006.02.006. MR 2284288

$\left[\mathrm{CCD}^{+}{ }^{13}\right]$ Eduardo Cattani, María Angélica Cueto, Alicia Dickenstein, Sandra Di Rocco, and Bernd Sturmfels, Mixed discriminants, Math. Z. 274 (2013), no. 3-4, 761-778, DOI 10.1007/s00209-012-1095-8. MR3078246

[CDR08] Cinzia Casagrande and Sandra Di Rocco, Projective $\mathbb{Q}$-factorial toric varieties covered by lines, Commun. Contemp. Math. 10 (2008), no. 3, 363-389, DOI 10.1142/S0219199708002818. MR2417921 
[DDRM18] Alicia Dickenstein, Sandra Di Rocco, and Ralph Morrison, Iterated multivariate discriminants, manuscript.

[DDRP09] Alicia Dickenstein, Sandra Di Rocco, and Ragni Piene, Classifying smooth lattice polytopes via toric fibrations, Adv. Math. 222 (2009), no. 1, 240-254, DOI 10.1016/j.aim.2009.04.002. MR.2531373

[DEK14] Alicia Dickenstein, Ioannis Z. Emiris, and Anna Karasoulou, Plane mixed discriminants and toric Jacobians, SAGA - Advances in ShApes, Geometry, and Algebra, Geom. Comput., vol. 10, Springer, Cham, 2014, pp. 105-121, DOI 10.1007/978-3-31908635-4_6. MR3289657

[DFS07] Alicia Dickenstein, Eva Maria Feichtner, and Bernd Sturmfels, Tropical discriminants, J. Amer. Math. Soc. 20 (2007), no. 4, 1111-1133, DOI 10.1090/S0894-0347-07-005620. MR 2328718

[DN10] Alicia Dickenstein and Benjamin Nill, A simple combinatorial criterion for projective toric manifolds with dual defect, Math. Res. Lett. 17 (2010), no. 3, 435-448, DOI 10.4310/MRL.2010.v17.n3.a5. MR.2653680

[DNV12] Alicia Dickenstein, Benjamin Nill, and Michèle Vergne, A relation between number of integral points, volumes of faces and degree of the discriminant of smooth lattice polytopes (English, with English and French summaries), C. R. Math. Acad. Sci. Paris 350 (2012), no. 5-6, 229-233, DOI 10.1016/j.crma.2012.02.001. MR2911928

[DR06] Sandra Di Rocco, Projective duality of toric manifolds and defect polytopes, Proc. London Math. Soc. (3) 93 (2006), no. 1, 85-104, DOI 10.1017/S0024611505015686. MR 2235483

[EG15] Alexander Esterov and Gleb Gusev, Systems of equations with a single solution, J. Symbolic Comput. 68 (2015), part 2. 116-130, DOI 10.1016/j.jsc.2014.09.007. MR.3283858

[Est10] Alexander Esterov, Newton polyhedra of discriminants of projections, Discrete Comput. Geom. 44 (2010), no. 1, 96-148, DOI 10.1007/s00454-010-9242-7. MR2639821

[Est18] Alexander Esterov, Characteristic classes of affine varieties and Plücker formulas for affine morphisms, J. Eur. Math. Soc. (JEMS) 20 (2018), no. 1, 15-59, DOI 10.4171/JEMS/758. MR3743235

[Est19] Alexander Esterov, Galois theory for general systems of polynomial equations, Compos. Math. 155 (2019), no. 2, 229-245, DOI 10.1112/s0010437x18007868. MR3896565

[FI16] Katsuhisa Furukawa and Atsushi Ito, A combinatorial description of dual defects of toric varieties, Commun. Contemp. Math., DOI 10.1142/S0219199720500017.

[For19] Jens Forsgård, Defective dual varieties for real spectra, J. Algebraic Combin. 49 (2019), no. 1, 49-67, DOI 10.1007/s10801-018-0816-4. MR3908355

[GKZ94] Israel M. Gel'fand, Mikhail M. Kapranov, and Andrei V. Zelevinsky, Discriminants, resultants, and multidimensional determinants, Mathematics: Theory \& Applications, Birkhäuser Boston, Inc., Boston, MA, 1994. MR.1264417

[Hov78] Askold G. Hovanskiॅ,, Newton polyhedra, and the genus of complete intersections (Russian), Funktsional. Anal. i Prilozhen. 12 (1978), no. 1, 51-61. MR487230

[HRS00] Birkett Huber, Jörg Rambau, and Francisco Santos, The Cayley trick, lifting subdivisions and the Bohne-Dress theorem on zonotopal tilings, J. Eur. Math. Soc. (JEMS) 2 (2000), no. 2, 179-198, DOI 10.1007/s100970050003. MR.1763304

[Ito15] Atsushi Ito, Algebro-geometric characterization of Cayley polytopes, Adv. Math. 270 (2015), 598-608, DOI 10.1016/j.aim.2014.11.010. MR.3286545

[Nil20] Benjamin Nill, The mixed degree of families of lattice polytopes, Ann. Comb. 24 (2020), no. 1, 203-216, DOI 10.1007/s00026-019-00490-3. MR4078146

[Pie15] Ragni Piene, Discriminants, polytopes, and toric geometry, Mathematics in the 21st century, Springer Proc. Math. Stat., vol. 98, Springer, Basel, 2015, pp. 151-162, DOI 10.1007/978-3-0348-0859-0_9. MR_3298234 
Fakultät für Mathematik, Otto-von-Guericke-Universität Magdeburg, UniversitätsPlatz 2, 39106 Magdeburg, Germany

Email address: christopher.borger@ovgu.de

Fakultät für Mathematik, Otto-von-Guericke-Universität Magdeburg, Universitätsplatz 2, 39106 Magdeburg, Germany

Email address: benjamin.nill@ovgu.de 